\title{
Nuevos registros de piojos (Insecta: Phthiraptera) en aves domésticas y ornamentales en Chile
}

\author{
New records of lice (Insecta: Phthiraptera) from domestic and \\ ornamental birds from Chile \\ D González-Acuña ${ }^{a^{*}}, \mathbf{J}$ Lara $^{\mathrm{a}}$, A Cicchino ${ }^{\mathrm{b}}$

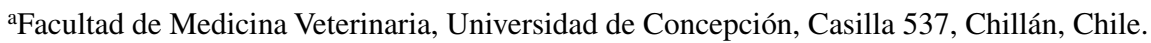

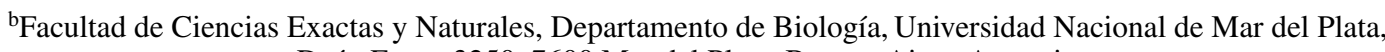 \\ Deán Funes 3250, 7600 Mar del Plata, Buenos Aires, Argentina.
}

\begin{abstract}
SUMMARY
The objective of this study was to confirm and update previous records of Phthiraptera in domestic and ornamental birds of Chile, through the examination of the plumage of the birds. The presence of Menopon gallinae, Goniodes gigas, Goniocotes gallinae and Menacanthus stramineus species on the domestic fowl Gallus gallus domesticus was confirmed, and the Menacanthus pallidulus is reported for the first time in Chile. In ornamental birds, seven species of lice were reported for the first time in Chile: M. stramineus in wild Turkey (Meleagris gallopavo), Goniodes pavonis and Amrysidea minuta in the Indian Peafowl, Goniocotes chrysocephalus and Oxylipeurus colchicus in the Common Pheasant (Phasianus colchicus), and Anaticola anseris and Trinoton anserinum in the Greylag Goose (Anser anser).
\end{abstract}

Palabras clave: aves, piojos, Phthiraptera.

Key words: birds, lice, Phthiraptera.

\section{INTRODUCCIÓN}

Los ectoparásitos en las aves juegan un papel importante en la dinámica poblacional, diversidad genética y resistencia natural de muchas especies animales (Villatoro y Sáenz 2005). Los piojos (Insecta: Phthiraptera) son uno de los taxa más frecuentes, se alimentan de descamaciones cutáneas, plumas, secreciones sebáceas y/o sangre (Cicchino y Castro 1998). Los reportes de piojos en aves domésticas en Chile se restringen a los trabajos de Roman (1947), Tagle (1953, 1966) y Artigas (1994).

La revisión de Alcaíno y Gorman (1999) de los parásitos en animales domésticos en Chile considera solamente los piojos reportados por Tagle (1966) en la gallina doméstica Gallus gallus domesticus (Linnaeus, 1758), sin haber en Chile hasta la fecha reportes precisos en otras especies de aves domesticadas por el hombre, por lo que la presente nota tiene como objetivo actualizar el estado de conocimiento de los Phthiraptera en aves domésticas y ornamentales de los órdenes Galliformes y Anseriformes en el país.

\section{MATERIAL Y MÉTODOS}

Entre los años 2000 y 2007 se colectaron piojos de las siguientes aves: doce gallinas domésticas Gallus gallus

\footnotetext{
Aceptado: 24.12.2008.

* Facultad de Medicina Veterinaria, Universidad de Concepción, Casilla 537, Chillán, Chile; danigonz@udec.cl
}

domesticus (Linnaeus 1758) procedentes de Chillán (VIII Región 36³6'S; 72²6'W), tres pavos, Meleagris gallopavo (Linnaeus 1758) procedentes de Chillán y Rancagua (VI Región $34^{\circ} 10^{\prime} \mathrm{S} ; 70^{\circ} 43^{\prime} \mathrm{W}$ ), seis pavos reales, Pavo cristatus (Linnaeus 1758) de Chillán, dos faisanes comunes, Phasianus colchicus (Linnaeus 1758), uno de Chillán y otro de Pinto (VIII Región 36² 2 'S; 7153’W), y dos gansos domésticos, Anser anser (Linnaeus 1758) de Chillán. Los piojos fueron colectados directamente de las aves y depositados en alcohol 70\%; posteriormente, cada ejemplar fue clarificado con $\mathrm{KOH}$ al $20 \%$ durante 12 a 24 horas, luego fue dejado por 24 horas en agua destilada, a continuación fueron deshidratados mediante soluciones ascendentes de alcohol (40\%, 70\% y 96\%) por 5 a 10 minutos en cada solución, para luego ser depositados en aceite de clavo durante 24 horas. Finalmente, cada ejemplar fue montado en Bálsamo de Canadá (Palma 1978, Price y col 2003), rotulado y observado bajo microscopio óptico para determinar su sexo, estado de desarrollo y especie usando las descripciones y claves de Clay $(1938,1940)$, Hohorst (1942), Emerson (1954), Eichler y Vasjukova (1980, 1981), Scharf y Price (1983), Tendeiro (1989).

\section{RESULTADOS Y DISCUSIÓN}

La gallina doméstica es una de las especies de aves en la que se ha colectado la mayor cantidad de especies de piojos, contabilizándose hasta la fecha según Price y col (2003) un total de doce. De ellas, siete han sido 
registradas en Chile, cuatro reconfirmadas en el presente reporte (cuadro 1) y la especie Menacanthus pallidulus (Neuman 1912) que es hallada en la presente investigación por primera vez en Chile. Las especies aún no citadas para Chile son principalmente de distribución holártica o bien de regiones tropicales: Lagopoecus sinensis (Sugimoto 1930), Lipeurus tropicalis (Peters 1931), Oxylipeurus dentatus (Sugimoto 1934) y Menacanthus cornutus (Shömmer 1913). No obstante, puede atribuirse también a la falta de muestreos exhaustivos de gallinas en territorio chileno, ya que esta última especie ha sido citada en Argentina (Cicchino y Castro 1998) como una de las especies más abundantes en distintas regiones del norte de la provincia de Buenos Aires. Kahn y col (2003) mencionan a Menacanthus stramineus (Nitzsch 1818), Menopon gallinae (Linnaeus 1758), Goniodes gigas (Taschenberg 1879), Goniocotes gallinae (De Geer 1778), Lipeurus tropicalis (Peters 1931), Lipeurus caponis (Linnaeus 1758) y Cuclutogaster heterographus (Nitzsch (en Giebel) 1866) como las especies más comúnmente aisladas en aves de corral. Curiosamente, la especie L. tropicalis no es reconocida en el compendio de Price y col (2003) como parásito de la gallina doméstica.

Roman (1947) encuentra Goniocotes rectangulatus (Nitzsch (en Giebel) 1866), cuyo hospedero tipo es Pavo cristatus, en la gallina doméstica, por lo que se asume que esta fue una transgresión parasitaria. Lo mismo sucede con la especie Chelopistes meleagridis (Linnaeus 1758), cuyo hospedero tipo es el pavo, M. gallopavo, no señalada aún en Chile en esta ave de producción y colectada por esta autora en la gallina. Ninguna de estas dos especies ha sido incorporada a la lista de los piojos de la gallina doméstica compendiada por Price y col (2003) porque estos autores no incluyeron registros causados por contaminaciones artificiales. También menciona $C$. heterographus en gallina, también reportada por Tagle $(1953,1966)$ en este hospedero, y Menopon trigonocephalum (Olfers 1816), que es un sinónimo absoluto de $M$. gallinae, citada en otras oportunidades para la gallina doméstica, y hallándose en al menos quince especies diferentes de aves galliformes (Price y col 2003). Junto a M. stramineus son las dos especies más frecuentemente reportadas en gallinas domésticas en diversos estudios en el mundo (ver Ancona 1935a , 1935 , Zayas 1941, Séguy 1944, Emerson 1951, Ansari 1955, Zlotorzycka y col 1974, Trivedi y col 1991, 1992, George y col 1992, Stewart y col 1996, Cicchino y Castro 1998, Amin-Babjee y col 1998, Permin y col 2002, Martín Mateo 2002, Kahn y col 2003, Ferrero y col 2004).

En el pavo doméstico, M. gallopavo, se colectó la especie $M$. stramineus, piojo que ha sido reportado en siete especies diferentes de aves (Price y col 2003), incluyendo Pavo cristatus (Silva y col 2004, Price y Graham 1997), hospedero en el cual no fue colectada aún esta especie en Chile.

Roman (1947) reporta Ch. meleagridis, como ya se señaló, en la gallina, de manera que la carencia de citas sobre pavo obedece al número reducido de colectas que a la fecha se han realizado sobre este hospedero en Chile.

Cuadro 1. Listado de publicaciones en aves domésticas y ornamentales en Chile en donde se indica la especie de Phthiraptera colectada, localidad y la fuente.

List of reports made on domestic and ornamental birds of Chile indicating the species of Phthiraptera collected, locality and source.

\begin{tabular}{|c|c|c|c|}
\hline Hospedador & Phthiraptera & Localidad & Autor \\
\hline Gallus gallus domesticus & $\begin{array}{l}\text { Menopon gallinae } \\
\text { Goniodes dissimilis } \\
\text { Goniodes gigas } \\
\text { Goniocotes gallinae } \mathrm{C}, \mathrm{D} \\
\text { Menacanthus stramineus } \\
\text { Menacanthus pallidulus } \\
\text { Lipeurus caponis }_{\text {Cuclogaster heterographus }}^{\mathrm{G}} \\
\text { Goniocotes rectangulatus } \\
\text { Chelopistes meleagridis }^{\mathrm{H}}\end{array}$ & $\begin{array}{l}\text { Chillán, no se indica } \\
\text { No se indica } \\
\text { Chillán, no se indica } \\
\text { Chillán, Chimbarongo } \\
\text { Chillán } \\
\text { Chillán } \\
\text { Valdivia } \\
\text { No se indica } \\
\text { No se indica } \\
\text { No se indica }\end{array}$ & $\begin{array}{l}1,2,3,4 \\
2 \\
1,2,3,4 \\
1,2,3,4 \\
1,2,3,4,5 \\
1 \\
2,3,4,6 \\
2,3,4 \\
2 \\
2\end{array}$ \\
\hline Melegris gallopavo & Menacanthus stramineus & Rancagua & 1 \\
\hline Pavo cristatus & $\begin{array}{l}\text { Goniodes pavonis } \\
\text { Amrysidea minuta }\end{array}$ & Chillán & 1 \\
\hline Phasianus colchicus & $\begin{array}{l}\text { Goniocotes chrysocephalus } \\
\text { Oxylipeurus colchicus }\end{array}$ & Pinto & 1 \\
\hline Anser anser & $\begin{array}{l}\text { Anaticola anseris } \\
\text { Trinoton anserinum }\end{array}$ & Bulnes & 1 \\
\hline
\end{tabular}

A: Roman (1947) la menciona como Menopon trigonocephalum. B Roman (1947) la menciona como Goniocotes gigas. C Tagle (1966) la menciona como Gonoides hologaster. D Tagle (1966) la menciona como Goniocotes hologaster. E: Roman (1947) lo menciona como Menopon biseriatus. F: Tagle (1966) la menciona como Eomenacanthus stramineus. G: Tagle (1966) lo menciona como Lipeurus heterographus. H: Roman (1947) la menciona como Goniodes stilifer.

Autores: 1. Presente estudio; 2. Roman, 1947; 3. Tagle, 1953, 4. Tagle, 1966; 5. Artigas, 1994. 6. Torres, 1974. 
En el $100 \%$ de los pavos reales examinados $(\mathrm{n}=6)$ se aislaron las especies Amrisidea minuta y Goniodes pavonis, dos de las doce especies de piojos que se han reportado en esta ave en distintas partes del mundo (Price y col 2003). $G$. pavonis es específica de $P$. cristatus, mientras que $A$. minuta se halla en las dos especies del género Pavo, P. cristatus y P. muticus (Price y col 2003). Roman en 1947 menciona a G. rectangulatus (Nitzsch (en Giebel) 1866) en gallina doméstica, por lo que valen las mismas consideraciones hechas para C. meleagridis.

En los dos faisanes (P. colchicus) procedentes de la localidad de Pinto se colectaron las especies Goniocotes chrysocephalus (Giebel 1874) y Oxylipeurus colchicus (Clay 1938), que son dos de las 11 especies mencionadas para este hospedero. G. chrysocephalus ha sido sólo citada en dos especies de fasiánidos: Bonasa umbellus (Linnaeus 1766) y $P$. colchicus; por otro lado, O. colchicus es un piojo que ha sido descrito sólo en $P$. colchicus (Price y col 2003).

En el ganso común (A. anser) se colectaron Anaticola anseris (Linnaeus 1758) y Trinoton anserinum (Fabricius 1805), dos de las siete especies descritas en esta ave (Price y col 2003). La especie A. anseris ha sido citada en diez especies hospedadoras diferentes; sin embargo, A. anser es el hospedero tipo y ha sido frecuentemente mencionada parasitando esta especie (Séguy 1944, Blagoveshchensky 1948, Tuleshkov 1958). Por otro lado, la especie T. anserinum ha sido determinada en nueve especies de aves de los géneros Anser y Cygnus (Price y col 2003), siendo A. anser su hospedero tipo. Llama la atención no haber colectado en A. anser las especies Anatoecus dentatus (Scop 1763) y A. icterodes (Nitzsch 1818), especies frecuentes en muchas especies de aves de la familia Anatidae (64 y 72 hospedadores respectivamente) en distintas regiones del mundo (Cicchino y Castro 1998, Price y col 2003), además de ser especies ya citadas en la XII Región de Chile en caiquenes (Chloephaga picta) (González-Acuña y col 2005) y en Anas georgica de la VII y VIII (Hinojosa 2007).

La presente nota es una contribución a la actualización del conocimiento de los Phthiraptera en aves domésticas y ornamentales de los ordenes Galliformes y Anseriformes en el país, sin embargo un aumento en los muestreos, así como una mayor dispersión de las localidades de colección, seguramente incrementará la cantidad de taxa descritos para Chile.

\section{RESUMEN}

Por medio del examen del plumaje de aves domésticas y ornamentales en Chile se reconfirma la presencia de los phthiraptera Menopon gallinae, Goniodes gigas, Goniocotes gallinae y Menacanthus stramineus en la gallina doméstica, Gallus gallus domesticus y se registra además por primera vez en el país la especie Menacanthus pallidulus. En aves ornamentales se registran en pavo, Meleagris gallipavo, la especie $M$. stramineus, en pavo real, Pavo cristatus las especies Goniodes pavonis y Amrysidea minuta, en faisán común Phasianus colchicus las especies Goniocotes chrysocephalus y Oxylipeurus colchicus y en ganso doméstico Anser anser las especies Anaticola anseris y Trinoton anserinum. Los siete registros realizados en aves ornamentales representan nuevos reportes para Chile.

\section{REFERENCIAS}

Alcaíno H, T Gorman. 1999. Parásitos de los animales domésticos en Chile. Parasitol al Día 23, 33-41.

Amin-Babjee S, CC Lee, AA Mahmood. 1998. Prevalence of ectoparasite infestation in different age groups of village chickens. $J$ Vet Malaysia 9, 50-60.

Ancona L. 1935 a Contribución al conocimiento de los piojos de los animales de México. II. Menopon gallinae Linn. An Inst Biol Mex 6, 53-62.

Ancona L. 1935 ${ }^{\text {b }}$. Contribución al conocimiento de los piojos de los animales de México. III. Goniocotes hologaster Nitzsch. An Inst Biol Mex 6, 119-128.

Ansari M. 1955. Synoptic table for the determination of Mallophaga infesting the domestic fowl (Gallus gallus domesticus). Indian $J$ Entom 17, 245-270.

Artigas J. 1994. Entomología Económica. Insectos de interés agrícola, forestal, médico veterinario (Nativos, introducidos y susceptibles de ser introducidos). Ediciones Universidad de Concepción. Vol. 1 Pp 935-963.

Blagoveshchensky D. 1948. Mallophaga's ptits Barakinskikh ozer (I). Parasit Sb Zoolog Ins Ak Nauk SSSR 10, 259-294.

Cicchino A, D Castro. 1998. Amblycera En: Morrone J, Coscarón S. Biodiversidad de artrópodos argentinos, una perspectiva biotaxonómica. Ediciones Sur, La Plata, Argentina, Pp 84-107.

Clay T. 1938. Revision of the genera and species of Mallophaga occurring on Gallinaceous hosts. Part I. Lipeurus and related genera. Proc Zool Soc Lon (Series B) 108, 109-204.

Clay T. 1940. Genera and species of Mallophaga occurring on Gallinaceous hosts. Part II. Goniodes. Proc Zool Soc Lond (Series B) 110, 1-120.

Eichler W, TT Vasjukova. 1980. Die Mallophagengattung Anaticola (Phthiraptera, Mallophaga). Dtsch Entomol Z 27, 335-375.

Eichler W, TT Vasjukova. 1981. Die Mallophagengattung Trinoton. Mitt Zool Mus Berlin 57, 23-62.

Emerson K. 1951. A list of Mallophaga from Gallinaceous birds of North America. J Wildlife Manage 15, 193-195.

Emerson 1954. A review of the genus Menopon Nitzsch, 1818 (Mallophaga). Ann Mag Nat Hist 7, 225-232.

Ferrero A, MM Gutiérrez, SH García, D Castro. 2004. Phthiraptera (Arthropoda, Insecta) en Gallus gallus (Galliformes, Phasianidae) en criaderos de áreas urbanas y suburbanas de la Ciudad de Bahía Blanca, Provincia de Buenos Aires, Argentina. Entomología y Vectores 11, 297-303,

George JB, S Otobo, J Ogunleye, B Adediminiyi. 1992. Louse and mite infestation in domestic animals in northern Nigeria. Trop Anim Health Prod 24, 121-124.

González-Acuña D, O Skewes, C Candia, R Palma, L Moreno. 2005. Estudio del parasitismo gastrointestinal y externo en caiquén Chloephaga picta Gmelin, 1789 (Aves, Anatidae) en la región de Magallanes, Chile. Parasitol Latinoam 60, 86-89.

Hinojosa A. 2007. Especificidad por hospedador de ecto y endoparásitos metazoos de Anas georgica Gmelin, 1789 (Aves: Anseriformes) y su relación con la prevalencia e intensidad de infección. Tesis Magíster, Universidad de Concepción, Concepción, Chile.

Hohorst W. 1942. Wenig bekannte Hühner-Mallophagen. Senckenbergiana $25,222-232$.

Khan MN, M Nadeem, I Zafas, MS Sajid, RZ Abbas. 2003. Lice infestation in poultry. Int J Agricult Biol 5, 213-216.

Martin Mateo MP. 2002. Mallophaga, Amblycera. En: Ramos MA, Alba JT, Bellés XR, Gosálbes JN, Guerra AS, Macpherson EM, Martín FP, Serrano JM, Templado JG (eds). Fauna Ibérica. Vol 20. Museo Nacional de Ciencias Naturales (CSIC), Madrid, España.

Palma R. 1978. Slid-mounting of lice: a detailed description of the Canada balsam technique. N Z Entomol 6, 432-436.

Permin A, JB Esmann, CH Hoj, T Hove, S Mukaratirwa. 2002. Ecto-, endo- and haemoparasites in free-range chickens in the Goromonzi District in Zimbabwe. Prev Vet Med 54, 213-224. 
Price RD, OH Graham. 1997. Chewing and sucking lice as parasites of mammals and birds. Technical Bulletin Number 1849. Agric Res Serv 1849, 1-257.

Price RD, RA Hellenthal, RL Palma, KP Johnson, DH Clayton. 2003. The chewing lice: world checklist and biological overview. Illinois Natural History Survey, Special Publication 24. Illinois, USA.

Roman R. 1947. Ecto y endoparásitos de las gallinas. Revista Chilena de Historia Natural 53, 3-55.

Séguy E. 1944. Insectes ectoparasites. Faune de France. Paul Lechevalier, Paris, France.

Scharf WC, RD Price. 1983. Review of the Amryrsidea in the subgenus Argimenopon (Mallophaga: Menoponidae). Ann Ent Soc Amer 76, 441-451.

Silva SO, HH de Oliveira, R Hidalgo Friccielo, NM Serra-Freire. 2004. Malófagos parasitas de aves campestres cativas do zoológico municipal Quinzinho de Barros, Sorocaba, Estado de São Paulo, Brasil. Entomologia y Vectores 11, 333-339.

Stewart RK, F Clark, M Petrie. 1996. Distribution of chewing lice upon the polygynous peacock Pavo cristatus. J Parasitol 82, 370-372.

Tagle I. 1953. Parásitos de los animales domésticos en Chile determinados en el Instituto de Investigaciones Veterinarias. Agric Tec 13, 93-108.
Tagle I. 1966. Parásitos de los animales domésticos en Chile. Bol Chil Parasitol 21, 118-121.

Tendeiro J. 1989. Études sur les Mallophages (Insecta, Mallophaga) des Galliformes de 1'Afrique centrale. II - Genre goniocotes Burmeister, 1838. García de Orta, Serie Zoologia 15, 63-97.

Trivedi MC, BS Rawat, AK Saxena. 1991. The distribution of lice (Phthiraptera) on poultry (Gallus domesticus). Int J Parasit 21, 247-249.

Trivedi MC, AK Saxena, BS Rawat. 1992. Incidence of Mallophaga on poultry in Dehradun (India). Angew Parasitol 33, 69-78.

Tuleshkov K. 1958. Pukhojady-Mallophaga po widnite ptitsy ot razredite Gressores i Pygopodes v Blgaria. Izvest na Zool Inst Sofia 7, 195-217.

Villatoro F, J Sáenz. 2005. La fragmentación del hábitat-Impactos sobre la dinámica huésped-parásito de la avifauna en paisajes agropecuarios de Esparza, Costa Rica. Boletín de la Asociación Ornitológica de Costa Rica 9, 3-10.

Zayas F. 1941. Los malofagos de las aves domésticas en Cuba. Memorias de la Sociedad Cubana de Historia Natural 15, 201-210.

Zlotorzycka J, W Eichler, HW Ludwig. 1974. Taxonomie und Biologie der Mallophagen und Läuse mitteleuropäischer Haus- und Nutztiere. Parasit Schrift Reihe 22, 1-160. 